\title{
Counterpossible Reasoning in Physics
}

\author{
Alastair Wilson \\ University of Birmingham \& Monash University \\ a.j.wilson@bham.ac.uk
}

23rd February 2021

Abstract: This paper explores three ways in which physics may involve counterpossible reasoning. The first way arises when evaluating false theories: to say what the world would be like if the theory were true, we need to evaluate counterfactuals with physically impossible antecedents. The second way relates to the role of counterfactuals in characterizing causal structure: to say what causes what in physics, we need to make reference to physically impossible scenarios. The third way is novel: to model metaphysical dependence in physics, we need to consider counterfactual consequences of metaphysical impossibilities. Physics accordingly bears substantial and surprising counterpossible commitments. 


\section{Introduction}

It is a truism that physics involves reasoning about alternative physical possibilities. To explain and predict actual observations, we construct models of the phenomena which represent alternative physically possible histories; perhaps we also assign a probability distribution over these histories. This essentially modal character of physics has been emphasized by authors as varied as Sellars, Suppes, Cartwright, Ladyman and Ross, and Maudlin. Does physics also involve reasoning about physical impossibilities? This question has received much less attention.

Why suspect that physics requires us to reason about the physically impossible? It is tempting to think that physicists need have no truck with impossible scenarios, given their narrow focus on predicting actual phenomena. However, there are at least three powerful reasons to think that the broader explanatory project of physics will incur non-trivial commitments concerning physical impossibilities: an argument from physical theorizing (\$3), an argument from causal structure $(\$ 4)$ and an argument from grounding structure (\$5).

Although the argument from physical theorizing can be resisted in several ways, the arguments from causal structure and grounding structure are more robust. There are two main ways to respond to these latter arguments: either deny that physics tells us about causal/grounding structure, or accept that physics incurs substantive metaphysical commitment in the form of patterns of non-trivial counterpossible counterfactuals. Neither option will be attractive to scientific realists who take sceptical or deflationary attitudes to metaphysics, and so the argument of this paper raises a new puzzle for those scientific realists. 
The argument from physical theorizing threatens only the necessitarian view that the fundamental laws of physics are non-contingent. It leaves untouched contingentists who acknowledge metaphysical possibilities featuring different fundamental laws of physics. However, I shall argue that every view of the modal status of fundamental phsyics needs an adequate response to the argument from grounding structure, and once such a response is in place then necessitarians can use it to resolve their problem with physical theorizing. One conclusion of this paper is accordingly that nomic necessitarianism is in better shape than is generally appreciated.

I begin in the next section $(\$ 2)$ with a brief survey of views on the modal status of laws of physics, and summarize reasons for thinking that these laws are metaphysically necessary. Orthodoxy in metaphysics says that physical laws are metaphysically contingent, but there is little support for this orthodoxy beyond intuition and precedent. The most persuasive reason usually given to favour contingentism is some version of the argument from physical theorizing (\$3). I argue that necessitarians have plausible responses to this argument. But necessitarians are not out of the woods; I next present the argument from causal structure $(\$ 4)$ and show that it escapes all the necessitarian responses to the argument from physical theorizing. Necessitarianism can in the end be rehabilitated, however, by consideration of the argument from grounding structure (\$5), which poses a puzzle for necessitarians and contingentists alike. The upshot $(\$ 6)$ is that necessitarianism and contingentism are on a par with respect to considerations of counterpossible reasoning in physics. Scientific realists, whatever their view on the modal status of fundamental physics, need to take on some substantial commitments concerning the impossible. 


\section{The Modal Status of Fundamental Physics}

As already noted, it is commonplace to regard fundamental physics as modally rich: our best physical theories describe a space of physical possibilities. The main controversy in the area concerns how physical modality relates to other forms of modality, in particular to metaphysical modality. The usual contingentist view is that the physical possibilities are a proper subset of the metaphysical possibilities. The contrary necessitarian view is that the physical possibilities just are the metaphysical possibilities, at least in so far as sense can be made of metaphysical possibility at all.

In my terminology, necessitarians include both those who take the notion of metaphysical modality seriously and identify it with physical modality, and those who are sceptical of the notion of metaphysical modality and recognize physical possibility as the most permissive form of objective possibility. While the sceptics might resist the 'necessitarian' label, I think this is more a verbal dispute than anything else; the disagreement boils down to the question of whether physical necessity plays enough of the relevant theoretical role to deserve the title 'metaphysical necessity'. In any case, this disagreement is moot here. Sceptics and deflationists concerning the notion of metaphysical necessity are still subject to the arguments in $\$ 3-5$, and so I classify sceptical and deflationary approaches as necessitarian for present purposes.

There is a wrinkle in the literature on the modal status of laws of nature that needs to be mentioned then set aside. The debate over the modal status of laws is entangled with a tangential debate over identity criteria for physical properties. Contrast the question of whether an inverse-cube law of attraction could hold with the question of whether an inverse-cube law 
of attraction could govern the behaviour of the specific property mass. The first question concerns what patterns of nomic behaviour are possible; the second question concerns which possible patterns can count as patterns of instantiation of mass. Schaffer (2005) distinguishes two resultant grades of necessitarianism:

Nomic necessitarianism: Properties obey the same laws of nature in every world in which they exist.

Modal necessitarianism: The laws of the actual world are the laws of all possible worlds.

Modal necessitarianism is the version on which I shall focus in this paper; from now on I shall drop the qualifier. Thus necessitarianism involves the identification of physical and metaphysical possibility, with the consequence that anything that is not in fact physically possible is also metaphysically impossible. This is the consequence which seems to conflict most directly with more familiar applications of counterpossible reasoning in physics. Ultimately, though, we shall see in $\$ 5$ that even giving up necessitarianism does not accommodate the full range of apparent counterpossibles in physics. Attributions of grounding structure to the physical world entail counterfactuals such that implementing their antecedents violates not merely laws of physics but also laws of metaphysics.

Why might one incline towards necessitarianism? I have already mentioned a sceptical/deflationary attitude towards metaphysical modality, which is often associated with deep suspicion of the method of relying on conceivability to establish possible cases; clear examples are Ladyman and Ross (2007) and Callender (2011). If you think metaphysical modality is an 
invention of analytic metaphysicians who took Kripke rather too seriously, then you are unlikely to think that there is any interesting objective sense in which the laws of nature could have been different. Of course, you might still think that other laws of nature are 'logically possible', but that is close to platitudinous: nobody thinks false physical theories are always inconsistent. What is at issue is what really can bappen, and this is not settled by logic alone.

Necessitarianism permits us to characterize objective modality in straightforwardly physical terms: the space of objective metaphysical possibilities is just the state space of the true fundamental physical theory. There need be no distracting 'alien' properties which present problems of ineffability, no hard questions about a fundamental language in which the space of possibilities is characterized. The fundamental physical properties are all the fundamental properties that there can be, and we may characterize modal space directly using our best theories in fundamental physics. If the actual world is wholly constituted by some quantum fields, then what it is to be an objective possibility is to be some possible state of those quantum fields. This is the first advantage of necessitarianism; it enables us to bring to bear the descriptive resources of fundamental physics to characterize objective modality generally and exhaustively. Contingentists need to look elsewhere for an account of their range of possible systems of physics.

This descriptive advantage of necessitarianism over contingentism is linked to an epistemic advantage. Necessitarians need no sui generis modal epistemology; instead they can co-opt the epistemology of fundamental physics to map the extension of objective modal space. By identifying fundamental physical theories (or at least, our best guesses at them), we can 
identify the space of objective possibilities (or at least, our best guess at it). This advantage is significant, since modal epistemology as a subfield is currently something of a disaster area. The main recognized method involves inferring possibility in key cases based on intuitions of conceivability; this method finds its canonical formulation in Chalmers (2002). Despite widespread unease about this method even within metaphysics, the lack of any systematic plausible alternative ensures ongoing reliance upon it; Schaffer (2005), for example, defends it as the only viable account on offer despite its problems. Necessitarianism provides our badly-needed alternative route to knowledge of objective possibility: physics itself. The resulting modal epistemology is simple, unmysterious, and naturalistic.

I will briefly mention two further arguments, following Schaffer (2005) and Wilson (2013). The first argument highlights the rational relevance of laws: necessitarianism explains, where contingentism cannot, why we care about the laws of nature. Necessitarianism likewise explains, where contingentism cannot, why we hold laws of nature fixed in the large majority of our counterfactual reasoning. These arguments both turn on the less-is-more nature of necessitarianism; since there are no objective possibilities in which laws of nature are violated, then i) knowing the laws enables us to focus attention on possible outcomes and ii) holding fixed laws in our ordinary counterfactual reasoning prevents our being distracted by impossibilities.

With this clarification of and preliminary case for necessitarianism in place, it is time for my first argument concerning counterpossible reasoning in physics: the argument from physical theorizing. 


\section{The Argument from Physical Theorizing}

Physics is difficult, and false theories abound. To have any hope of identifying the correct fundamental theory - or even of making progress towards it - we need to be able to theorize: we need to be able to think critically about multiple theories and to evaluate them by contrasting their different consequences. What are we doing when we assess a given theory's consequences? On one very familiar reading, what we are doing is asking counterfactual questions about what would be the case if the theory in question were correct. If quantum chromodynamics were correct, quark jets would be seen by the LHC - and so they are. Imminently I shall discuss some alternatives to this 'counterfactual consequence' account of physical theorizing; but first I shall use the account to formulate the argument from physical theorizing.

Suppose some string-theory model of quantum gravity is correct. Then necessitarianism makes these counterfactuals into counterpossibles:

A. If spacetime were Newtonian, it would have Euclidean geometry. $[\mathrm{T}]$

B. If general relativity were complete and correct, gravity would be quantized. [F]

C. If loop quantum gravity were correct, there would be no spin foams. $[\mathrm{F}]$

Reasoned evaluation of the theories in question seems to require us to ascribe these counterfactuals non-trivial truth-values. This is the argument from physical theorizing: 
1. Evaluating Newtonian mechanics/general relativity/loop quantum gravity involves assessing the (non-trivial) truth or falsity of counterfactuals A/B/C.

2. We can and do evaluate Newtonian mechanics/general relativity/loop quantum gravity in our physical theorizing.

3. A/B/C are counterfactuals with physically impossible antecedents.

4. We assess the (non-trivial) truth or falsity of counterfactuals with physically impossible antecedents in our physical theorizing. (From $1,2,3$.

Contingentists can readily accept 4 , but it spells trouble for necessitarians. In a necessitarian picture, there are too few objective possibilities to underwrite the needed variation in truth-values for the counterfactuals in question.

There are two main schematic responses which necessitarians can give to the argument from physical theorizing. They can offer a non-trivial semantics for counterfactuals with physically impossible antecedents (counternomics), for example by appealing to impossible worlds or by ingeniously repurposing possible worlds, and thereby give non-trivial truthconditions to counternomics. That would amount to a reconciliation of 4 with necessitarianism. Alternatively, they can deny 1 and give an explanation of the apparent epistemic role of counternomics in physical theorizing that is compatible with their triviality. I recommend that necessitarians adopt the latter approach, since it retains the following principle: 
Counterfactual Aboutness: Counterfactuals are about how things stand with respect to genuine alternative possibilities.

Counterfactual Aboutness is associated with the influential semantics for counterfactuals proposed by Lewis (1973) and by Stalnaker (1968). Though not entailed by the semantics, the principle forms part of a simple and appealing approach to counterfactuals and modality incorporating the semantics. It also fits neatly with the principles linking counterfactuals to metaphysical modality in Williamson (2007). Rather than delve into the literature on counterfactuals, I will take Counterfactual Aboutness as axiomatic and focus on responses to the argument from physical theorizing which retain it. Accordingly, I will not here explore potential applications of impossible-worlds theory to physical theorizing, although this is undoubtedly a worthwhile project.

Let us focus then on premise 1, and distinguish some different ways in which it might be denied. I will discuss four types of response: twodimensionalist, metatheoretic, error-theoretic, and fictionalist. Although I will only be able to present each of these in barest outline and will not attempt a systematic assessment, my discussion ought to provide a sense of the range of options available.

The two-dimensionalist response draws on the distinction between indicative and subjunctive readings of the relevant conditionals. In particular, it evaluates counterpossible counterfactuals as embedded in indicative conditionals (Handfield 2004). Consider the following contrasting pair: 
- If contingentism is correct, then if spacetime were Newtonian it would have Euclidean geometry.[T]

- If contingentism is correct, then if general relativity were complete and correct, gravity would be quantized. [F]

By embedding the problematic counterpossible inside a suppositional context with respect to which it is not after all a counterpossible, we 'suppose away' the problem; relative to the supposition of contingentism, the counterfactuals are not counterpossibles and hence the embedding conditionals can have the variation in truth-value which is required if they are to play their intended role with respect to physical theorizing. However, the two-dimensionalist solution is unappealing. It complicates the semantics of the relevant counterpossibles, it complicates the behaviour of counterpossibles when embedded within broader patterns of reasoning, and it implausibly makes the evaluation of counternomics parasitic on a false metaphysical theory, contingentism.

A more popular approach is to go metatheoretic. The metatheoretic response to the argument from counterpossible reasoning embraces triviality for counternomic counterfactuals, but denies that physical theorizing needs to appeal to counterfactuals. Counternomic evaluation is replaced by direct theorizing about models. For example,

$\mathrm{N}$ If spacetime were Newtonian, it would have a Euclidean geometry.

becomes

N* Models of Newtonian spacetime assign it a Euclidean geometry. 
While the metatheoretic response has some attractions - theorists are already committed to the relevant facts about models to which the response appeals - it also has some substantial downsides. Since it is not always clear whether some counterfactual is a counternomic, it will not always be clear whether it is a genuine counterfactual or a disguised description of models. But the underlying problem is that the metatheoretic approach simply does too much damage to logical form. $\mathrm{N}$ is about spacetime and its geometry. It is not about theories or models; reinterpreting it in such a way is ad hoc, and ought to be a last resort. (This failure of the metatheoretic approach recapitulates the failure of formalism in philosophy of mathematics.) Fortunately, better responses are available.

A more plausible relative of the metatheoretic response is an errortheoretic response. According to the error theory, when doing physics we reason non-trivially with counternomics but we err in so doing: there are in fact no non-trivial counternomic facts out there to vindicate our non-trivial counternomic reasoning. Nonetheless, error theorists can offer a systematic explanation of why we succeed in our theoretical goals despite this central false presupposition: the relevant metatheoretic fact about models explains why our practice of assigning non-trivial truth-values to counternomic counterfactuals succeeds regardless of our error. I think the error-theoretic response is more attractive than the metatheoretic response, but I still regard it as ad hoc and sub-optimal, for the broadly charity-hased reasons that make error theories unattractive more generally. Although the error theory is a viable strategy for a deflationary account of counterpossible theory evaluation, still it would be better not to have to rely on it.

The fictionalist response has much in common with the error-theoretic response, but improves upon it by making better rational sense of our 
practice of theory-evaluation. According to the fictionalist about counternomics, when doing exploratory physics we typically make and evaluate counternomic counterfactuals within the context of a pretence: we pretend that the antecedent (along with any theoretical background which it contextually presupposes) is a genuine physical possibility. What I am proposing here is a fictionalism about counternomics in the make-believe style of Walton (1990), not the fictional-operator fictionalism of Rosen (1990). Fictionality is not a truth-conditional operator attaching to sentential contents, but something more akin to a speech-act or pragmatic performative. The pretence may or may not reflect genuine possibility; but (importantly) we do not need to know whether it does accurately reflect genuine possibility in order to engage in the pretence.

The fictionalist response has all of the advantages of the error-theoretic response, without convicting the practice of physics of systematic confusion. Counterfactual Aboutness is retained, both inside and outside the scope of the pretence. And it is antecedently plausible that physical theorizing does enmesh the theorizer in a certain kind of pretence. In order to take seriously a theory as a candidate for truth, one does have to - at least temporarily - shelve concerns about its potential impossibility and explore it as presumptively possible.

Here a helpful analogy may be drawn with reductio arguments in mathematics. Since (at least classically) mathematical statements are true if possible, then in order to reason non-trivially about false mathematical claims one must be able to reason non-trivially about the impossible. Mathematicians may use a reductio argument to establish the falsity of a claim which they already know to be false (for example, when teaching students); in order to do this they must be able to temporarily adopt the 
pretence that the claim in question is true, and hence possible. This pretence is often marked in mathematics by the phrase 'suppose for reductio'; in non-mathematical contexts, 'suppose for the sake of argument' seems to play the same role.

As always with fictionalist proposals, we can ask why the fiction is a useful one. Some fictionalisms founder on this challenge; for example, modal fictionalists struggle to explain why a fiction of concrete possible worlds should be a useful one. However, fictionalists about impossible theory-evaluation in physics (as in mathematics) can offer a plausible schematic account of the usefulness of the fiction. It is not always evident to an inquirer whether a given theory (physical or mathematical) is possible or impossible. So, for exploratory purposes, we may give it a temporary benefit of the doubt. There are scenarios - pedagogical or historical, for example - where it is useful to be able to reason about the theory's consequences while bracketing the question of its possibility. This is what a fictionalist interpretation of counterpossible reasoning in physics models reasoners as doing.

An aside: Kimpton-Nye (2020) defends a version of fictionalism about counternomics although he develops the view differently; Kimpton-Nye's counternomic fictionalism builds 'according to [the] fiction' (2020, 530) directly into the semantics for counternomics, and also fails to preserve Counterfactual Aboutness.

All the components for the main arguments of this section are now in place. Necessitarians face a serious challenge, the objection from physical theorizing, but they can meet the challenge: deflationary accounts of the epistemic role of counterpossibles in physical theorizing are compatible 
with Counterfactual Aboutness. In particular, we don't need to soberly judge hypothetical scenarios as objectively possible in order to investigate them and the prospective theories that characterize them. We can (and I think physicists, like mathematicians, do) adopt a non-committal pretence of possibility for the sake of the argument. This provides us with a suitably deflationary picture of the counterpossibles involved in physical theory evaluation, and it disarms the threat that necessitarianism faces from the argument from physical theorizing.

\section{The Argument from Causal Structure}

Up to this point we have attended to the role of counterpossibles in the practice of physical theorizing: the task is to identify the correct theory. A very different way in which counterpossibles might be needed is to capture causal structure: the task is then to explicate the theory's consequences for the causal structure of the world. In order to focus the discussion, I will presuppose an interventionist approach to causation. Interventionists analyse causal claims in terms of the counterfactual consequences of interventions: causal relationships hold only if certain counterfactuals are true, where the antecedents of these 'interventionist counterfactuals' specify that a suitable intervention occurs. These interventions may be physically impossible (Woodward 2003), and so from the necessitarian point of view intervention counterfactuals may be counterpossibles. If intervention counterfactuals trivialize, then we lack the variation in truthvalue to support attributions of non-trivial causal structure to reality. This is the argument from causal structure in a nutshell; like the argument from physical theorizing, it poses a challenge for necessitarians. 
A first problematic kind of impossible intervention is a conservationlaw-violating intervention. If the Sun were removed from the solar system by an intervention, the Earth would cease to move in an ellipse. So the presence of the Sun is the cause of the Earth's elliptical motion. Making sense of this causal claim requires an intervention to remove the Sun. The Sun cannot simply be deleted from reality: this would violate conservation of energy and angular momentum.

A second, even more problematic, class of impossible interventions involves changing the background structure of the physical world. If an intervention were to adjust the number of spatial dimensions to four, then the orbits of the planets would be unstable. So, the three-dimensionality of space is the cause of the stable orbits of the planets. Making sense of this requires an intervention to alter the number of spatial dimensions. Intervening on the dimensionality of space is physically impossible.

Woodward himself presents the dimensionality case as an example of non-causal explanation, denying that there is any intervention that can explicate the dependence involved or help answer the relevant 'what-ifthings-had-been-different' question (Woodward 2003, 220). I am more inclined to see the case as causal in nature despite its unfamiliarity, but no matter; the previous example of the sun's orbit suffices to drive the argument from causal structure of this section. On Woodward's interpretation, the dimensionality example might instead illustrate my next argument from grounding structure $(\$ 5)$, or indeed might illustrate a distinct 'argument from non-causal structure'.

Necessitarians might try to apply one of the four responses explored in $\$ 3$ to the challenge from causal structure. However, in the present context 
these responses fail, since they undermine the objectivity of the resulting picture of reality's causal structure. A two-dimensionalist approach makes causal claims true only relative to the supposition that contingentism is correct. A metatheoretic approach captures only causal dependencies amongst models, not causal dependencies in reality. An error-theory approach leads to an error theory of the causal claims. A fictionalist approach leads to fictionalism about the causal claims. The key difference between the argument from physical theorizing and the argument from causal structure is that causal structure is part of the world as physics aims to reveal it, whereas physical theorizing is merely a part of the investigative process - a ladder which can be kicked away once the correct theory is identified. Consequently, the argument from causal structure presents a more serious problem for necessitarianism.

However, before finalizing a verdict on the necessitarianism vs contingentism debate, we should look again to the larger dialectical context. What we have here may be a symptom of a deeper problem, in which case necessitarians could be off the hook. To capture the full range of dependencies in nature, even contingentists about laws may have to tolerate non-trivial counterpossibles. This prospect is addressed - and endorsed in the next section.

\section{The Argument from Grounding Structure}

The deep structural similarity between grounding and causation suggests that the asymmetry of grounding is reflected in an asymmetry of consequences of interventions. If we intervene to change the ground fact, the grounded fact changes. If we intervene to change the grounded fact, 
the ground fact is unchanged. Grounding claims thus entail counterfactuals with metaphysically impossible antecedents, as I have argued elsewhere (Wilson 2018a, 2018b).

Consider the claim that the pressure of a gas is grounded in the average linear momentum of the gas molecules. If an (impossible) intervention were to ground an increased average momentum differently, perhaps by increasing the mass of the molecules while holding their trajectories fixed, pressure would increase along with momentum. But if an (impossible) intervention were to ground a higher pressure directly, perhaps by somehow amplifying the force of the impacts with the container sides, then the average momentum of the molecules would be unchanged. Making sense of this requires interventions which violate grounding principles linking molecular motion with pressure. The counterfactuals we need here are countermetaphysicals.

The literal truth of grounding claims is therefore in direct tension with Counterfactual Aboutness. A two-dimensionalist approach makes grounding claims true only relative to the supposition that metaphysics is contingent. A metatheoretic approach captures only grounding dependencies internal to models and not grounding dependencies in reality. An error-theory approach leads to an error theory of the grounding claims. A fictionalist approach makes the grounding claims literally false: the grounding claims hold only within the pretence, however useful or extensive that pretence may be. Fictionalism about grounding in general has defenders (e.g. Thompson 2018). But if grounding structure is sometimes correctly represented by (interpreted) physical theories, then deflationary solutions to the problem of physical theorizing remain vulnerable to the problem of grounding structure. 
The argument from grounding structure afflicts both contingentists and necessitarians. I see two potential escape routes: account for counterpossibles in terms of impossible worlds (sacrificing Counterfactual Aboutness), or deny objective grounding structure in the physical world. Only the latter option supports contingentism over necessitarianism; however, I take it that denying all grounding dependencies within physics is fairly radical. It conflicts with obvious truisms such as the claim that the temperature of an ideal gas is grounded in molecular motion, or that the centre of mass of a compound system is grounded in the masses and locations of its components.

\section{Conclusion}

This paper has argued that physics is enmeshed in counterpossible reasoning. What follows for the modal status of the laws of nature? Necessitarians have various options for responding to the argument from physical theorizing ( $(\$ 3)$. These responses fail to carry over to the argument from causal structure $(\$ 4)$, which does present a distinctive problem for necessitarianism. However, attention to the metaphysical dependencies invoked by physics (\$5) reveals that capturing non-contingent dependence is a problem for everyone. Considerations of practice in physics and of the content of our physical theories therefore provide no strong argument for contingentism over necessitarianism. This result is interesting and surprising in its own right, but it also enhances the overall prospects for 
necessitarianism. The laws of fundamental physics may yet turn out to possess, as Kripke put it, 'necessity in the highest degree' (1980, 99).*

* Thanks to Amanda Bryant, Nina Emery, Nick Emmerson, Joaquim Giannotti, Dana Goswick, Mike Hicks, Chris Hitchcock, Vera Hoffmann-Kolss, Mario Hubert, Noelia Iranzo Ribera, Matthias Jenny, Nick Jones, Sam KimptonNye, Dennis Lehmkuhl, Brian McLoone, Kristie Miller, Daniel Nolan, Martin Pickup, Alex Roberts, Miranda Rose, Katie Robertson, Gonzalo RodriguezPereyra, Chip Sebens, Henry Taylor, Jim Woodward, David Yates, and an anonymous referee for Philosophy of Science. Thanks also to audiences at the workshop on Counterpossibles, Counternomics, and Causal Theories of Properties in Köln, the FraMEPhys Workshop on Grounding and the Laws of Nature in Birmingham, the Caltech Philosophy of Physics Reading Group, and the reading group of the "Emergence in the Natural Sciences" project in Lisbon (PTDC/FER-HFC/30665/2017). This work forms part of the project A Framework for Metaphysical Explanation in Physics (FraMEPhys), which received funding from the European Research Council (ERC) under the European Union's Horizon 2020 research and innovation programme (grant agreement no. 757295). Funding was also provided by the Australian Research Council (grant agreement no. DP180100105). 


\section{References}

Callender, Craig. 2011. "Philosophy of Science and Metaphysics." In The Bloomsbury Companion to the Philosophy of Science, ed. Steven French and Juha Saatsi, 33-54. London: Bloomsbury.

Chalmers, David. 2002. "Does Conceivability Entail Possibility?”. In Conceivability and Possibility, ed. John Hawthorne and Tamar Gendler, 145-200. New York: Oxford University Press.

Handfield, Toby. 2004. "Counterlegals and Necessary Laws." The Philosophical Quarterly 54 (216): 402-419.

Kimpton-Nye, Samuel. 2020. "Necessary Laws and the Problem of Counterlegals." Philosophy of Science 87 (3): 518-535.

Kripke, Saul. 1980. Naming and Necessity. Cambridge: Harvard University Press.

Ladyman, James, and Don Ross. 2007. Every Thing Must Go. New York: Oxford University Press.

Lewis, David. 1973. Counterfactuals. Oxford: Blackwell.

Rosen, Gideon. 1990. “Modal Fictionalism.” Mind 99: 327-364.

Schaffer, Jonathan. 2005. “Quiddistic Knowledge.” Philosophical Studies 123 (1-2): 1-32.

Stalnaker, Robert. 1968. "A Theory of Conditionals." In Studies in Logical Theory, ed. Nicholas Rescher. Oxford: Blackwell.

Thompson, Naomi. 2018. "Irrealism about Grounding." Royal Institute of Philosophy Supplements 82: 23-44.

Walton, Kendall. 1990. Mimesis as Make-Believe. Cambridge: Harvard University Press. 
Williamson, Timothy. 2007. The Philosophy of Philosophy. Oxford: Blackwell. Wilson, Alastair. 2013. "Schaffer on Laws of Nature.” Philosophical Studies 164 (3): 653-667.

_ 2018a. "Grounding Entails Counterpossible Non-Triviality."

Philosophy and Phenomenological Research 96 (3): 716-728.

__ 2019b. "Metaphysical Causation.” Noûs 52 (4): 723-751, 2018.

Woodward, James. 2003. Making Things Happen. New York: Oxford University Press. 